\title{
Fluctuation-dissipation relations and energy landscape in an out-of-equilibrium strong glass-forming liquid
}

\author{
Antonio Scala ${ }^{1}$, Chantal Valeriani ${ }^{1}$, Francesco Sciortino ${ }^{1,2}$ and Piero Tartaglia ${ }^{1,2}$ \\ 1 Dipartimento di Fisica e Istituto Nazionale per la Fisica della Materia, \\ Universitá di Roma La Sapienza, P.le Aldo Moro 2, I-00185, Roma, Italy \\ ${ }^{2}$ INFM, Center for Statistical Mechanics and Complexity, Universitá di Roma La Sapienza
}

(27 Sept 2002)

\begin{abstract}
We study the out-of-equilibrium dynamics following a temperature-jump in a model for a strong liquid, BKS-silica, and compare it with the well known case of fragile liquids. We calculate the fluctuation-dissipation relation, from which it is possible to estimate an effective temperature $T_{\text {eff }}$ associated to the slow out-of-equilibrium structural degrees of freedom. We find the striking and unexplained result that, differently from the fragile liquid cases, $T_{\text {eff }}$ is smaller than the bath temperature.
\end{abstract}

PACS numbers: 61.20.Lc,64.60.My,61.43.Fs 
A central issue of statistical physics is to link the static properties of a thermodynamical system to its dynamics. A fundamental result in this direction has been the derivation of the fluctuation-dissipation theorem (FDT) [1], which links the response functions to autocorrelation functions; however, derivations of the FDT require the system to be in equilibrium and the response to be in linear regime.

Recently, a great deal of work has concentrated on the generalization of the FDT to out-of-equilibrium (OOE) situations [2]; in particular, it has been proposed to generalize FDT in the form [3]

$$
R_{A B}\left(t, t^{\prime}\right)=\frac{X\left(C_{A, B}\left(t, t^{\prime}\right)\right)}{k_{B} T} \frac{\partial C_{A B}\left(t, t^{\prime}\right)}{\partial t^{\prime}}
$$

where $A$ and $B$ are conjugated observables, $R_{A B}\left(t, t^{\prime}\right)$ is the mutual response of the observable $A$ to an impulsive conjugated field applied at $t^{\prime}, C_{A B}\left(t, t^{\prime}\right)=\left\langle A(t) B\left(t^{\prime}\right)\right\rangle-\langle A(t)\rangle\left\langle B\left(t^{\prime}\right)\right\rangle$ is the unperturbed correlation function of the observables and $T$ is the temperature of the bath; $X$ is supposed to be a function of $C_{A B}$ only and equals to one in equilibrium and linear regime. In recent analytical and numerical work, it has been observed that the out of equilibrium dynamics which follows a $T$-jump is characterized by an FDT relation with $X=1$ at short times and $X<1$ at long times [4]. It has been proposed to interpret the ratio $T_{\text {eff }}=T / X$ as an effective temperature associated to the different timescales of the system [5]: while fast degrees of freedom are supposed to be thermalised so that $T_{\text {eff }}$ equals the bath temperature $T$ (and hence $X=1$ and ordinary FDT holds), slow degrees of freedom are still reminiscent of the temperature of the system before being brought in OOE so that $T_{e f f} \neq T$ (i.e. $X \neq 1$ ). In general, it is observed that for quenching experiments $T_{\text {eff }}>T$ for the slow degrees of freedom; also in the case of a "reverse" quench, where the system is suddenly heated up, slow degrees of freedom "remember" the initial state being $T_{\text {eff }}<T$ [6]. For fragile liquids, the results are consistent with the one step replica symmetry breaking (1RSB) scenario both for quenches [4,7] and for "crunches" [8] (sudden changes of volume); in this scenario, $X$ becomes a step function equal to one at short times $\left(T_{\text {eff }}=T\right)$ and equal to a constant less than one $\left(T_{\text {eff }}>T\right)$ at long times. Exceptions to this behavior are pointed out in refs. [9-11].

The slow dynamics of supercooled systems have been considered also under a complimentary approach, based on the statistical properties of the free-energy [12] or the potential energy [13] landscape. In the landscape approach the free energy of a system is separated in configurational contributions, linked to the basins of attraction of local minima of the landscape, and in vibrational contributions linked to the number of states in such basins. It has been suggested that fast relaxations are related to the exploration of states inside a basin, while slow relaxations correspond to the exploration of different potential energy landscape basins. The topology of the landscape explored should therefore correlate to the dynamics of the system. Support of this interpretation for fragile liquids has been presented both for equilibrium [14] and out-of-equilibrium conditions [15].

Under the assumption that the basins explored in OOE are the same that would be explored in equilibrium [16], it is possible to extend the landscape approach to OOE situations at the expenses of adding an additional parameter to the thermodynamic formalism. In OOE, the landscape-based liquid free energy can be written as [7]

$$
f\left(e_{i s}, R\right)=e_{i s}-T_{\text {int }} s_{\text {conf }}\left(e_{i s}\right)+f_{\text {vib }}\left(e_{i s}, T\right)
$$


where $e_{i s}$ is the average energy of the local potential energy minima (the so called "inherent structures" [13]), the configurational entropy $s_{\text {conf }}$ counts the number of minima of energy $e_{i s}, f_{v i b}$ is the average free energy of a basin of depth $e_{i s}, T$ is the temperature of the bath and $T_{i n t}$ is a Lagrange multiplier that extremizes $f$; in the equilibrium case, $T_{i n t}$ is just the temperature of the bath $T$. The extremum condition $\partial f / \partial e_{i s}=0$ gives an expression of the internal temperature $T_{\text {int }}$

$$
T_{i n t}\left(e_{i s}, T\right)=\left(1+\frac{\partial f_{v i b}\left(e_{i s}, T\right)}{\partial e_{i s}}\right) / \frac{\partial s_{\text {conf }}\left(e_{i s}\right)}{\partial e_{i s}}
$$

that can be calculated from the equilibrium values of $s_{\text {conf }}$ and from analytical approximations for $f_{v i b}$. In the case of a prototype fragile liquid, the binary mixture Lennard-Jones, it has been verified that in OOE conditions the configurational temperature $T_{\text {int }}$ calculated from the landscape approach and the effective temperature $T_{\text {eff }}$ measured from the extension of the FDT coincide [7]. This coincidence is supported from the analytical predictions for the prototype fragile mean field system, the p-spin model, for which again $T_{\text {int }}=T_{\text {eff }}[17]$.

In the case of strong liquids, the situation is less developed. Only recently, a realistic model for liquid silica, the BKS model [18], has been studied in detail in equilibrium conditions. At variance with fragile systems, where the $T$ dependence of the characteristic times is always super-Arrhenius, BKS-silica shows a high $T$ region $(T>3330 K)$ where the dynamics is super-Arrhenius and a low- $T$ region $(T<3300 K)$ where the equilibrium dynamics recovers an Arrhenius behavior. For $T>3330 K$, the dynamics of BKS silica is well described by mode-coupling theory; at difference with other fragile liquids, the presence of local tetrahedral structures and therefore of a strong oxygen-silicon-oxygen correlation, makes also three-point correlation functions to be taken in account in order to get quantitative agreement with the theory [19]. For $T<3330 K$, BKS silica becomes strong, in agreement with the behavior of real silica. Interestingly enough, despite this strong-to-fragile crossover, the structural relaxation times of BKS-silica are well described in the whole temperature region by the Adam-Gibbs formula [20] as in the case of fragile liquids; moreover, free directions in configuration space vanish at the mode-coupling temperature [21]. Therefore, landscape theory is able to rationalize the equilibrium dynamics of fragile and strong systems despite the alleged diversity of their landscapes [22].

It is therefore important to check if $T_{i n t}=T_{\text {eff }}$ also in the case of strong liquids: if this would be the case, we could have afforded a simple thermodynamic understanding of OOE liquids in terms of two temperatures, the kinetic temperature $T$ associated with the fast vibrational degrees of freedom and the internal temperature $T_{\text {int }}$ (or its dynamical counterpart $T_{\text {eff }}$ ) associated to the slowly evolving configurational degrees of freedom [23].

In our simulations we have employed the BKS-potential [18] with the parameters of ref. [20]. Simulations are for systems of $N=999$ particles $\left(N_{S i}=333\right.$ and $\left.N_{O}=666\right)$ at fixed density $\rho=2.36 \mathrm{~g} / \mathrm{cm}^{3}$ with a Nose'-Hoover thermostat. Long range forces have been calculated implementing the Ewald sums. We have quenched 280 independent configurations equilibrated at $T=3800 \mathrm{~K}$ (well above the fragile-to-strong crossover at $T=3330 \mathrm{~K}$ [24]) at two different final temperatures $T_{1}=2900$ and $T_{2}=2500$; both these temperatures are in the region where BKS-silica has a strong behavior [24]. The Nose'-Hoover parameters have been selected to bring the kinetic energy of the system in equilibrium within $0.5 \mathrm{ps}$. We have measured two unperturbed correlation functions of the form $\left\langle A\left(t+t_{w}\right) B\left(t_{w}\right)\right\rangle_{0}$, where $A$ and 
$B$ are conjugated observables with zero average in absence of a perturbation. Indicating with $\vec{r}_{l}^{b}$ the coordinates of the $l$-th oxygen atom $\left(l=1 . . N_{O}, N_{O}=666\right)$, the choice $A=\rho_{\text {coll }}^{o}=$ $\sum_{l}^{N_{O}} \exp \left(i \vec{k} \cdot \vec{r}_{l}^{o}\right) / \sqrt{N}$ and $B=\left(\rho_{\text {coll }}^{o}+\rho_{\text {coll }}^{o *}\right)$ corresponds to the dynamical structure factor $C_{\text {coll }}$; the choice $A=\rho_{\text {self }}^{o}=\exp \left(i \vec{k} \dot{\vec{r}}_{l}^{\rho}\right)$ and $B=\left(\rho_{\text {self }}^{o}+\rho_{\text {self }}^{o *}\right)$ corresponds to the self part of the intermediate scattering function $C_{\text {self }}$. In the case of the self correlation, averages over several ions have been performed. The total number of independent realizations (different configurations, different wave vectors, different ions) is $\sim 30000$ for the quenches at $T_{1}$ and $\sim 20000$ for the quenches at $T_{2}$, both for the self and for the collective case.

Fig. 1 shows the evolution of the average inherent structure energy during the aging, following the $T$-jump and compares it with the known equilibrium values. The average $e_{I S}$ decreases with time, while the system searches for deeper and deeper basins in the attempt to equilibrate. We note that the $e_{i s}$ decrease does not depend on the bath temperatures selected, suggesting that the system is exploring configuration space with a saddle-dominated entropic dynamics [25]. For example, after 2 ps, independently from the bath temperature, the system is exploring basins which are typically explored in equilibrium at $T \approx 3650 \mathrm{~K}$.

By calculating the density of states of the explored basins and comparing it with the corresponding quantity evaluated in equilibrium, we confirm that the basins explored during the aging dynamics have the same density of states of the basins explored in equilibrium. Since in the case of BKS-silica, the density of states does not depends on $e_{i s}$, in harmonic approximation $\partial f_{v i b} / \partial e_{i s}=0$. This implies that, according to Eq. 3, the $T_{i n t}$ of the BKSsystem when populating basins of average depth $e_{i s}$ coincides with the temperature at which basins of depth $e_{i s}$ are populated in equilibrium (Fig.1). It is therefore possible to calculate the internal temperature of the system inverting the equilibrium relations between $e_{i s}$ and $T$ [7] (Fig. 1). At the waiting time $t_{w}=2 p s$, we find that $T_{\text {int }} \approx 3650 K$ for both the quenches, so that $T_{\text {int }}>T_{1}, T_{2}$ and we expect to measure for the slow degrees of freedom a $T_{\text {eff }}$ higher than the bath temperature.

In order to measure $T_{\text {eff }}$, we evaluate the integrated response function $\chi=$ $V_{0} \int_{t_{w}}^{t} d t^{\prime} R\left(t, t^{\prime}\right)$; from Eq. 1 the slope $\frac{d \chi}{d C}$ of the parametric plot of $\chi$ versus $C$ yields $V_{0} \frac{X(C)}{k_{B} T}=-V_{0} \frac{1}{k_{B} T_{e f f}}$. We have applied conjugate fields $V_{0} B \theta\left(t-t_{w}\right)$ of constant amplitude $V_{0}$ after a waiting time $t_{w}=2 p s$, significatively bigger than the characteristic time of the microscopic dynamics (see Fig. 2), and measured the integrated responses $\chi_{\text {coll }}=\rho_{\text {coll }}^{o}$ and $\chi_{\text {self }}=\rho_{\text {self }}^{o}$. We have chosen $V_{0}=4.0 \mathrm{~kJ} / \mathrm{mol}$ for $B=\left(\rho_{\text {coll }}^{o}+\rho_{\text {coll }}^{o *}\right)$ and $V_{0}=5.0 \mathrm{~J} / \mathrm{mol}$ for $B=\left(\rho_{\text {self }}^{o}+\rho_{\text {self }}^{o *}\right)$ in order to have a good signal-to-noise ratio in the response function while being still well inside the linear regime region [28]. We have averaged response and correlation functions over 300 wave vectors of length $|\vec{k}|=28.6 \mathrm{~nm}^{-1}$ corresponding to the main peak of the static structure factor in order to have a good signal-to-noise ratio for the correlation functions [29]. We show in Fig. 2 the correlations and the response functions of the system. At the $t_{w}$ chosen, exploration of deeper basins has started as shown in Fig.1. This waiting time, which is much shorter than the infinite-time limit in which analytical predictions are derived, is consistent with the aging time chosen in most of previous studies of fragile liquids [30].

The parametric plots of $\chi$ versus $R$ for the quenches at the higher temperature $T_{1}$ are unusual (Fig. 3, left panels), in the sense that although we know the system is aging, the slope of the plots is consistent with a $T_{\text {eff }}=T$ along all the curves as in equilibrium FDT. 
This behavior is similar to the results of ref. [10] for the Fredrickson-Andersen model, but contrasts with our expectation of a $T_{\text {eff }}$ higher than the $T_{1}$. The parametric plots of $\chi$ versus $R$ at $T_{2}$ (Fig. 3, right panels) yields even more astonishing results: while at short times (high values of $\mathrm{C}$ ) the slope $\frac{d \chi}{d C}$ is equal to $-V_{0} \frac{1}{k_{B} T_{2}}$ yielding the usual equilibrium FDT relation, in the long time region (low values of $C$ ), the parametric plot bends upward, i.e. $T_{\text {eff }}$ is lower than the bath temperature.

In conclusion, we have shown that for a realistic model of strong liquid, the aging dynamics is not consistent with the commonly observed 1RSB scenario. Moreover, the behavior of the fluctuation-dissipation ratio $X$ contrasts with the possibility of interpreting $T_{\text {eff }}$ as a temperature. From the point of view of landscape theory, this effect could be due to a profound and still to be understood difference in the way strong and fragile landscapes are explored. As for strong liquids barriers to relaxation grow slower than for fragile liquids while exploring deeper regions of the landscape, the possibility remains in fact open that activated processes are "too fast" and don't allow to describe the system in terms of long-lived quasi-states [31] and to define therefore a simple extension of landscape thermodynamics to OOE conditions. The results reported in this letter clearly contradict the general consensus built in recent years in the study of models of fragile liquids $[4,7,8]$. This pushes for a deeper theoretical understanding of the behavior of the strong glass-forming liquids.

We acknowledge support from MURST PRIN 2000, INFM-PRA-HOP, INFM-Initiative Parallel Computing. 


\section{REFERENCES}

[1] H. B. Callen and T. A. Welton, Phys. Rev. 83, 34 (1951); R. Kubo, J. Phys. Soc. Jpn. 12, 570 (1957).

[2] L. F. Cugliandolo and J. Kurchan, Phys. Rev. Lett. 71, 173 (1993)

[3] L. F. Cugliandolo and J. Kurchan, J. Phys. A 27, 5749 (1994)

[4] G. Parisi, Phys. Rev. Lett. 79, 3660 (1997); E. Marinari, G. Parisi, F. Ricci-Tersenghi and J. J. Ruiz-Lorenzo, J. Phys. A:Math. Gen. 31, 2611 (1998); J. L. Barrat and W. Kob, Europhys. Lett. 46, 637 (1999)

[5] L. F. Cugliandolo, J. Kurchan and L. Peliti, Phys. Rev. E 55, 3898 (1997)

[6] L. Berthier, P. C. W. Holdsworth and M. Sellitto, J. Phys. A:Math. Gen. 34, 1805 (2001)

[7] F. Sciortino and P. Tartaglia, Phys. Rev. Lett. 86, 107 (2001)

[8] R. Di Leonardo, L. Angelani, G. Parisi, and G. Ruocco Phys. Rev. Lett. 84, 6054 (2000)

[9] A. Crisanti , F. Ritort, A. Rocco, and M. Sellitto, J. Chem. Phys. 113, 10615 (2001);

J. P. Garrahan and M. E. J. Newman, Phys. Rev. E 62, 7670 (2000)

[10] A. Buhot and J. P. Garrahan, Phys. Rev. Lett. 88, 225702 (2002)

[11] "Origins of the violation of fluctuation-dissipation theorem is systems with activated dynamics" A. Perez-Madrid, D. Reguera and J.M. Rubi, preprint cond-mat/0210089

[12] R. G. Palmer, Adv. In Phys. 31669 (1982)

[13] F. H. Stillinger and T. A. Weber, Phys. Rev. A 25, 978 (1982); Science 225, 983 (1984)

[14] P. G. Debenedetti and F. H. Stillinger, Nature 410, 259 (2001)

[15] E. La Nave, S. Mossa and F. Sciortino Phys. Rev. Lett. 88, 225701 (2002)

[16] W. Kob, F. Sciortino and P. Tartaglia, Europhys. Lett. 49, 590 (2000)

[17] S. Franz and M. A. Virasoro, J. Phys. A 33, 891 (2000)

[18] B. W. H. van Beest, G. J. Kramer and R. A. van Santen, Phys. Rev. Lett. 64, 1955 (1990).

[19] F. Sciortino and W. Kob, Phys. Rev. Lett. 86, 648 (2001); W. Kob, M. Nauroth and F. Sciortino, J. Non-cryst. Sol. 307, 181 (2002)

[20] I. Saika-Voivod, P. H. Poole and F. Sciortino, Nature 412, 514 (2001)

[21] E. La Nave, H. E. Stanley and F. Sciortino, Phys. Rev. Lett. 88, 035501 (2002)

[22] C. A. Angell, B. E. Richards and V. Velikov, J. Phys. Condens. Matter 11, A75 (1999).

[23] This assertion should be limited to "not so far" from equilibrium systems, as it is expected that two parameters are not enough to describe in general OOE systems [12]; moreover, this is certainly not true for systems with infinite replica symmetry breaking $[32]$

[24] J. Horbach and W. Kob, Phys. Rev. B 60, 3169 (1999)

[25] With saddle-dominated entropic dynamics we mean that the system is exploring the landscape through accessible saddles that lead to lower energy minima; this search is entropic, i.e. activation is due to the probability of finding a direction toward lower energies. In this regime, temperature controls just the velocity of the trajectory in the configuration space and small differences in kinetic temperature are irrelevant on logaritmic scale. If kinetic temperature is too low, after a first phase of entropic search saddles are not accessible anymore and the system has to cross barriers to relax to lower minima; it is therefore expected a relevant dependence of the dynamics on the 
kinetic temperature. Such scenario has been proposed for a fragile glass-former in [26] and verified in [27].

[26] W. Kob, F. Sciortino and P. Tartaglia Europhys. Lett. 49, 590 (2000)

[27] R. Di Leonardo, L. Angelani, G. Parisi, G. Ruocco, A. Scala, F.Sciortino Phil. Mag. B 82, $163(2002)$

[28] We have checked that the response functions scaled by $V_{0}$ still collapse on each other for values of the field up to three times bigger than the ones employed in the simulations.

[29] The importance of a good signal-to-noise ratio stems from the incredible difficulty in obtaining good statistics for the response and the correlation functions. We have been forced to average over $\sim 10^{4}$ quenches for each data set in order to obtain data of good quality; this has meant one year of continuous running on a proprietary cluster of six Compaq Alpha and on eight nodes of the SGI-Origin at Cineca.

[30] Choosing $t_{w} 10$ times bigger would have requested simulations 10 times longer to observe the same amount of decorrelation (assuming that structural decorrelation times scale as $t_{w}$ ). This would have been unfeasible with present computer facilities. It could be achieved at the expenses of decreasing by 10 the number of independent realizations and hence increasing the noise level.

[31] G. Biroli and J. Kurchan, Phys. Rev. E 64, 016101 (2001)

[32] E. Marinari, G. Parisi, F. Ricci-Tersenghi and J. J. Ruiz-Lorenzo, J. Phys. A 31, 2611 (1998) 


\section{FIGURES}

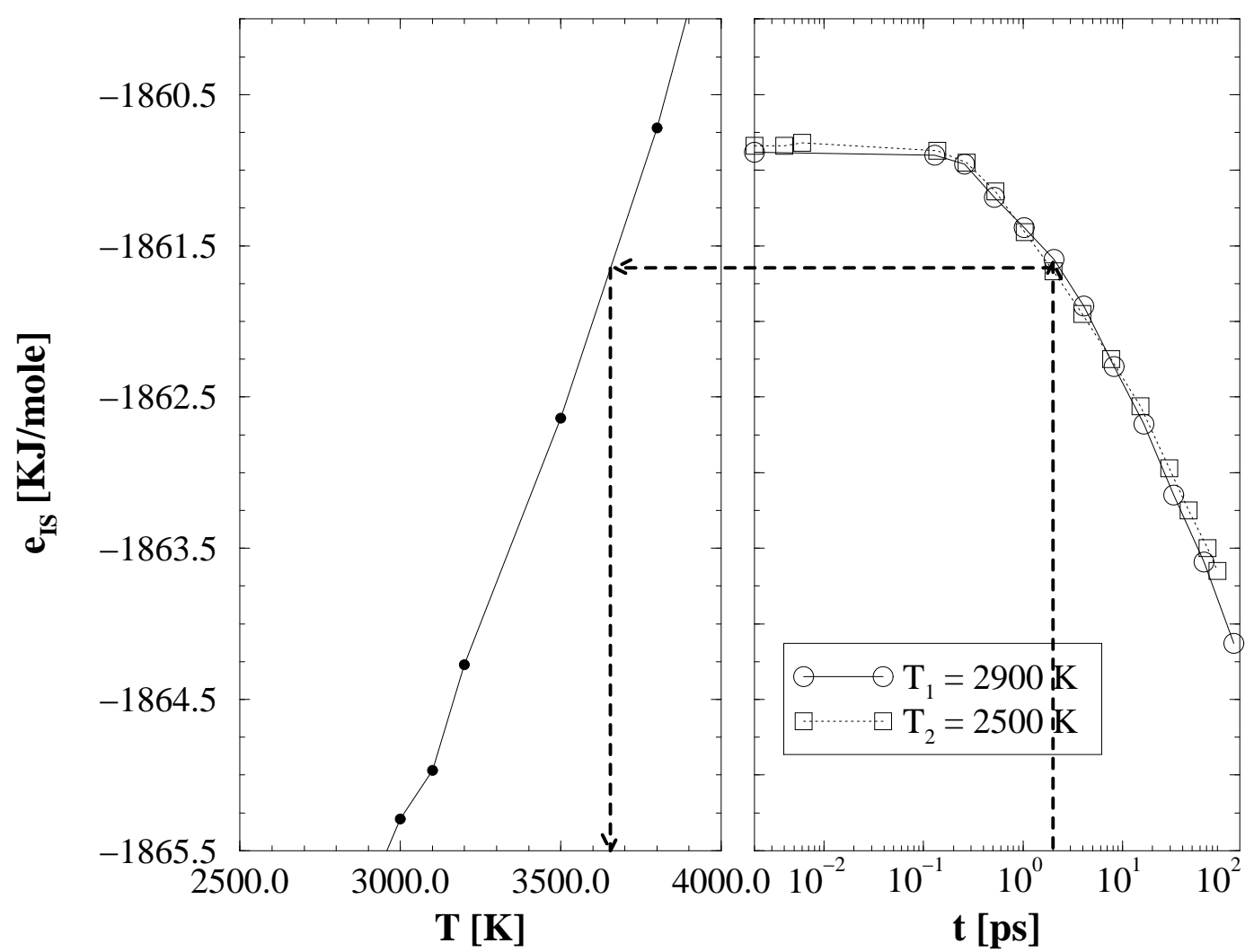

FIG. 1. Left panel: equilibrium relation between $e_{i s}$ and $T$. Right panel: $e_{i s}$ as a function of $t$ for the two quenches considered. The arrows show graphically the procedure that connects the value of $e_{i s}(t)$ to the equilibrium temperature $T\left(e_{i s}\right)$ for $t=t_{w}=2 p s$; landscape theory therefore predicts the temperature associated to the configurational degrees of freedom to be $T_{\text {int }} \approx 3650 \mathrm{~K}$ in both quenches. 

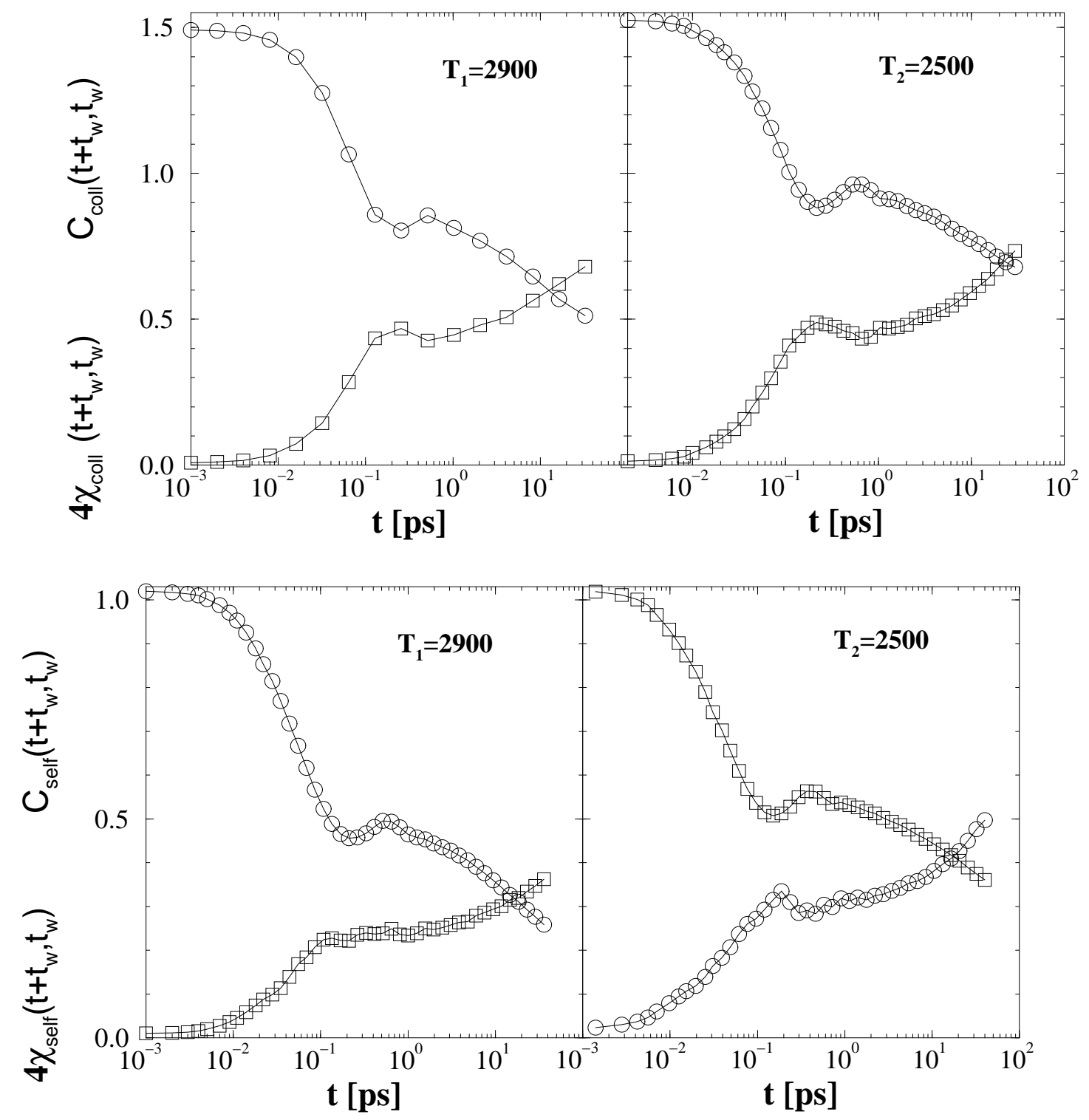

FIG. 2. Correlation and response functions for the collective (upper panels) and the self (lower panels) variables, at the two temperatures $T_{1}=2900 \mathrm{~K}$ (left panels) and $T_{2}=2500 \mathrm{~K}$. Short time behavior corresponds to the fast vibrational dynamics inside a basin; the corresponding first decay of the correlation functions to a plateau is fast. Long time behavior corresponds to the exploration of new minima causing the slow decay of correlation functions from the plateaus. 

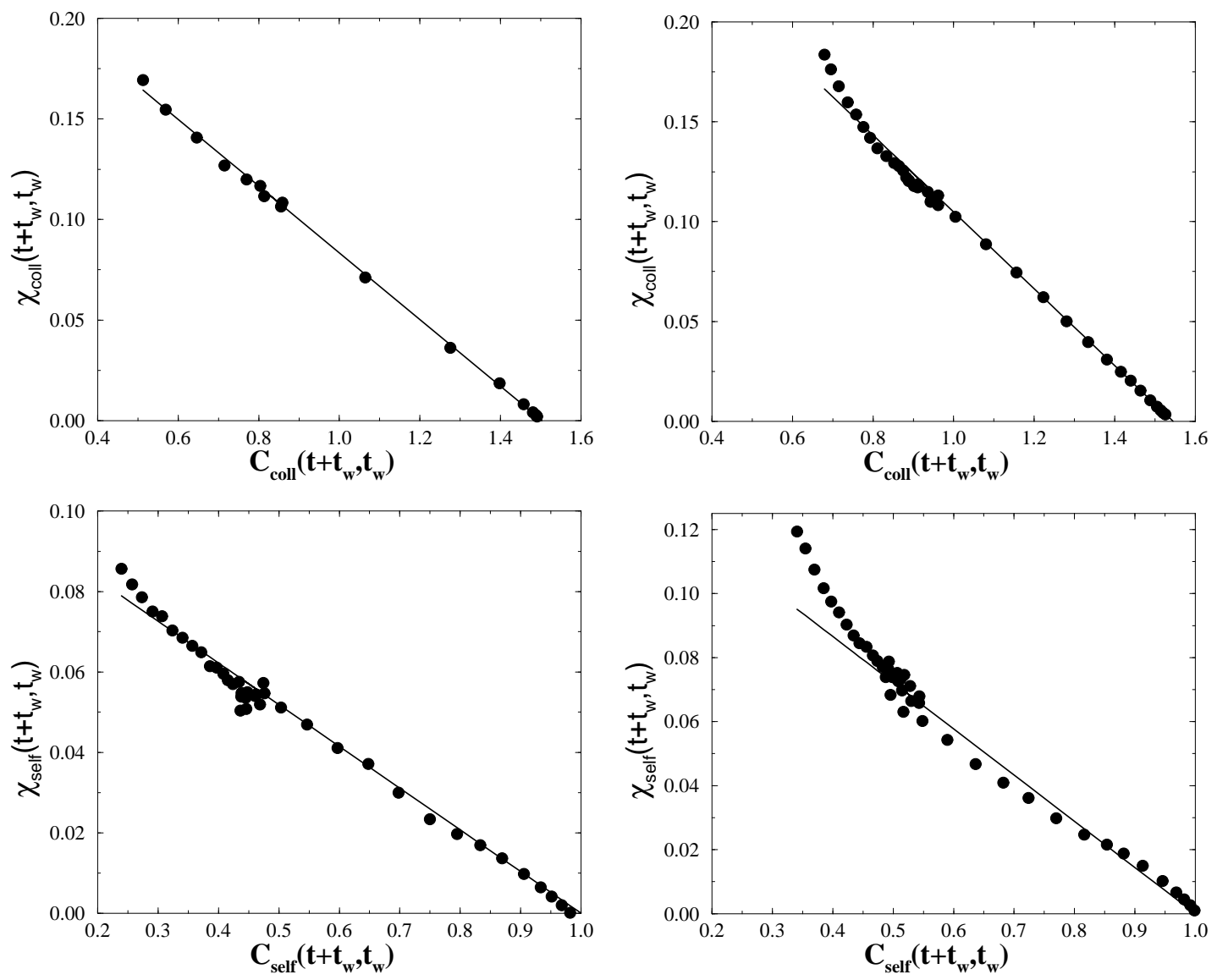

FIG. 3. Parametric plots of the correlation and response functions for the collective (upper panels) and the self (lower panels) variables, at the two temperature $T_{1}=2900 \mathrm{~K}$ (left panels) and $T_{2}=2500 \mathrm{~K}$; the slope $\frac{d \chi}{d C}$ is proportional to $-1 / T_{\text {eff }}$. Straight lines are the predictions of the equilibrium FDT theorem where the slope is proportional to $-1 / T$. At the lower temperature, $T_{\text {eff }}<T$ at long times (low values of correlation). 